\title{
On almost generalized 2-absorbing and weakly almost generalized 2-absorbing structures
}

\author{
Sirawich Chinwarakorn, Sajee Pianskool* \\ Department of Mathematics and Computer Science, Faculty of Science, Chulalongkorn University, \\ Bangkok 10330 Thailand
}

*Corresponding author, e-mail: sajee.pianskool.p.s@gmail.com

Received 20 May 2014

Accepted 17 Dec 2014

\begin{abstract}
In this paper, we define almost generalized 2-absorbing submodules (ideals) and weakly almost generalized 2-absorbing submodules (ideals). These are generalizations of the 2-absorbing and weakly 2-absorbing submodules (ideals) defined by Badawi and Darani. Their properties are investigated.
\end{abstract}

KEYWORDS: submodules

MSC2010: 13C99 13A15 13H05

\section{INTRODUCTION}

Throughout this paper, unless otherwise stated, all rings are commutative with non-zero identity and all modules over these rings are unitary. Let $R$ and $M$ stand for a ring and a module over ring $R$, respectively.

New objects related to prime and weakly prime ideals were introduced and studied by Badawi and Darani ${ }^{1}$. These are the concepts of 2-absorbing and weakly 2-absorbing ideals of commutative rings. Darani and Soheilnia ${ }^{2}$ introduced the concepts of 2-absorbing and weakly 2-absorbing submodules of modules over rings.

In module theory, it is known that the intersection of each pair of distinct prime submodules may no longer be a prime submodule. The intersection of each pair of distinct prime (weakly prime) submodules is a 2-absorbing (weakly 2-absorbing) submodule $^{2}$. Note also that primary submodules (ideals) are directly closed to prime submodules (ideals). These inspired us to define almost generalized 2-absorbing and weakly almost generalized 2-absorbing submodules (ideals) in order that the intersection of each pair of distinct primary (weakly primary) submodules (ideals) is an almost generalized 2-absorbing (weakly almost generalized 2-absorbing) submodule (ideal).

The rest of this paper contains four sections. The first section introduces almost generalized 2-absorbing and weakly almost generalized 2-absorbing structures. Almost generalized 2-absorbing structures are studied in two categories, namely, rings and modules. We provide proofs of some properties. In the second section, some results and some relations of almost generalized 2-absorbing submodules of a multiplication module are discussed, especially of a cyclic $R$-module. Then we investigate certain rings with the property that all proper ideals are weakly almost generalized 2-absorbing in the third section. In the last section, we study those concepts in decomposable commutative rings.

\section{AG2-ABSORBING AND WEAKLY AG2-ABSORBING STRUCTURES}

Definition 1 [Ref. 2] A proper submodule $N$ of $M$ is called a 2-absorbing (weakly 2-absorbing) submodule of $M$ if whenever $a, b \in R, m \in M$ and $a b m \in N(0 \neq a b m \in N)$, then $a m \in N$ or $b m \in N$ or $a b \in(N: M)$.

Definition 2 [Ref. 3] A proper ideal $I$ of $R$ is called a 2-absorbing (weakly 2-absorbing) ideal of $R$ if whenever $a, b, c \in R$ and $a b c \in I(0 \neq a b c \in I)$, then $a c \in I$ or $b c \in I$ or $a b \in I$.

Definition 3 [Ref. 4] A proper submodule $N$ of $M$ is called a primary (weakly primary) submodule of $M$ if whenever $r \in R, m \in M$ and $r m \in N(0 \neq r m \in N)$, then $m \in N$ or $r^{n} \in(N: M)$ for some $n \in \mathbb{N}$.

Definition 4 A proper ideal $P$ of $R$ is said to be a primary (weakly primary) ideal if whenever $a, b \in R$ and $a b \in P(0 \neq a b \in P)$, then $a \in P$ or $b^{n} \in P$ for some $n \in \mathbb{N}$. 
We extend these notions to almost generalized 2-absorbing and weakly almost generalized 2-absorbing submodules (ideals) as follows.

Definition 5 A proper submodule $N$ of $M$ is called an almost generalized 2-absorbing or AG2-absorbing (weakly almost generalized 2-absorbing or weakly AG2-absorbing) submodule of $M$ if whenever $a, b \in$ $R, m \in M$ and $a b m \in N(0 \neq a b m \in N)$, then $a^{i} m \in$ $N$ or $b^{j} m \in N$ or $(a b)^{k} \in(N: M)$ for some $i, j, k \in \mathbb{N}$.

A proper ideal $I$ of $R$ is called an almost generalized 2-absorbing or AG2-absorbing (weakly almost generalized 2-absorbing or weakly AG2-absorbing) ideal of $R$ if whenever $a, b, c \in R$ and $a b c \in I$ $(0 \neq a b c \in I)$, then $a^{i} c \in I$ or $b^{j} c \in I$ or $(a b)^{k} \in I$ for some $i, j, k \in \mathbb{N}$.

It is obvious from the above definition that the two AG2-absorbing (weakly AG2-absorbing) structures (in terms of submodules and ideals) are related, i.e., AG2-absorbing (weakly AG2-absorbing) ideals of a ring $R$ can be studied by considering the ring $R$ as an $R$-module. Note that the zero submodule (ideal) need not be an AG2-absorbing submodule (ideal) as the following example shows.

Example 1 By choosing $M=R=\mathbb{Z}_{30}$, we show that $\overline{2} \cdot \overline{3} \cdot \overline{5} \in\{\overline{0}\}$ but $\overline{2}^{i} \cdot \overline{5} \notin\{\overline{0}\}, \overline{3}^{j} \cdot \overline{5} \notin\{\overline{0}\}$ and $\overline{6}^{k} \notin$ $\{\overline{0}\}=\left(\{\overline{0}\}: \mathbb{Z}_{30}\right)$ for all $i, j, k \in \mathbb{N}$. Hence $\{\overline{0}\}$ is not an AG2-absorbing submodule of $\mathbb{Z}_{30}$.

The following example shows a difference between AG2-absorbing submodules and weakly AG2absorbing submodules. Clearly, every AG2-absorbing submodule is a weakly AG2-absorbing submodule but not vice versa in general.

Example 2 Consider the case where $R=\mathbb{Z}, M=$ $\mathbb{Z} / 42 \mathbb{Z}$ and $N=0$. Then $N$ is a submodule of $M$ and $2 \cdot 3 \cdot(7+42 \mathbb{Z})=0 \in N$ while $2^{i} \cdot(7+42 \mathbb{Z}) \notin N$, $3^{j} \cdot(7+42 \mathbb{Z}) \notin N$ and $(2 \cdot 3)^{k} \notin 42 \mathbb{Z}=(N: M)$ for all $i, j, k \in \mathbb{N}$. Hence the submodule $N$ is not AG2absorbing but it is weakly AG2-absorbing.

One can see that AG2-absorbing submodules are weakly AG2-absorbing submodules. Furthermore, it is obvious that every 2-absorbing (weakly 2-absorbing) submodule is an AG2-absorbing (weakly AG2absorbing) submodule of $M$. Likewise, it is clear that 2-absorbing submodules and weakly 2-absorbing submodules are generalizations of prime submodules and weakly prime submodules, and AG2absorbing submodules and weakly AG2-absorbing submodules are generalizations of primary submodules and weakly primary submodules. Similarly, these remarks also hold for ideals of the same kind. In other words, the word 'submodules' can be replaced by 'ideals.'

The intersection of each distinct pair of prime (weakly prime) submodules is 2-absorbing (weakly 2 -absorbing) ${ }^{2}$. This leads us to study the intersection of each distinct pair of primary (weakly primary) submodules in the following.

Theorem 1 (1) The intersection of each pair of primary submodules of $M$ (ideals of $R$ ) is an AG2absorbing submodule (ideal). (2) The intersection of each pair of weakly primary submodules of $M$ (ideals of $R$ ) is a weakly AG2-absorbing submodule (ideal).

Proof: (1) Let $N$ and $K$ be two distinct primary submodules of $M$. Moreover, let $a, b \in R$ and $m \in M$ be such that $a b m \in N \cap K$ but $a^{i} m \notin N \cap K$ and $b^{j} m \notin N \cap K$ for all $i, j \in \mathbb{N}$. Then we conclude that (a) for all $i \in \mathbb{N}\left(a^{i} m \notin N\right.$ or $\left.a^{i} m \notin K\right)$ and (b) for all $j \in \mathbb{N}\left(b^{j} m \notin N\right.$ or $\left.b^{j} m \notin K\right)$. Thus there are 4 cases to be considered:

(i) $a m \notin N$ and $b m \notin N$.

(ii) $a m \notin N$ and $b m \notin K$.

(iii) $a m \notin K$ and $b m \notin N$.

(iv) $a m \notin K$ and $b m \notin K$.

Case (i). Since $N$ is primary, $b(a m)=a b m \in N$ and $a m \notin N$, we show that $b^{n_{0}} \in(N: M)$ for some $n_{0} \in \mathbb{N}$. Thus $b^{k} m \in N$ for all $k \geqslant n_{0}$. It follows from the above conclusion (b) that $b^{k} m \notin K$ for all $k \geqslant n_{0}$ and then $b^{k} m \notin K$ for all $1 \leqslant k<n_{0}$. So $b^{k} \notin(K: M)$ for all $k \in \mathbb{N}$. Similarly, $a^{k} \notin(K: M)$ for all $k \in \mathbb{N}$. Since $K$ is primary, $a(b m) \in K$ and $a^{k} \notin$ $(K: M)$ for all $k \in \mathbb{N}$, it follows that $b m \in K$ which is a contradiction. Hence case (i) is not possible. Similarly, case (iv) is absurd.

Case (ii). Again, since $N$ is primary, $a m \notin N$ and $b m \notin K$, we show that $b^{n_{b}} \in(N: M)$ and $a^{n_{a}} \in(K$ : $M$ ) for some $n_{a}, n_{b} \in \mathbb{N}$. This yields

$$
\begin{aligned}
(a b)^{n_{a}+n_{b}}=a^{n_{a}+n_{b}} b^{n_{a}+n_{b}} & \in(K: M)(N: M) \\
\subseteq(N: M) & \cap(K: M)=(N \cap K: M) .
\end{aligned}
$$

Much as in case (ii), one can get that $(a b)^{k} \in(N \cap K$ : $M$ ) for some $k \in \mathbb{N}$ in case (iii). Hence we conclude that the intersection of each pair of distinct primary submodules of $M$ is AG2-absorbing as desired.

(2) The proof is similar to that of (1).

Another way to verify whether a proper submodule $N$ of $M$ is AG2-absorbing is to consider a particular submodule of the quotient $R$-module $M / N$. 
Proposition 1 Let $N$ and $K$ be submodules of $M$ with $K \subseteq N$. Then $N$ is an AG2-absorbing submodule of $M$ if and only if $N / K$ is an AG2-absorbing submodule of the R-module $M / K$.

Proof: Suppose first that $N$ is an AG2-absorbing submodule of $M$. Then $N / K$ is a proper submodule of the $R$-module $M / K$. Let $a, b \in R$ and $m \in M$ be such that $a b(m+K) \in N / K$. Then $a b m \in N$, so $N$ being an AG2-absorbing submodule gives $a^{i} m \in N$ or $b^{j} m \in N$ or $(a b)^{k} \in(N: M)$ for some $i, j, k \in \mathbb{N}$. Therefore $a^{i}(m+K) \in N / K$ or $b^{j}(m+K) \in N / K$ or $(a b)^{k} \in(N / K: M / K)$. Thus $N / K$ is an AG2absorbing submodule of $M / K$.

Conversely, assume that $N / K$ is an AG2-absorbing submodule of the $R$-module $M / K$. Then $N$ is a proper submodule of $M$. Suppose that $a, b \in R$ and $m \in M$ with $a b m \in N$. Then $a b(m+K) \in N / K$ so that $a^{i}(m+K) \in N / K$ or $b^{j}(m+K) \in N / K$ or $(a b)^{k} \in$ $(N / K: M / K)$ for some $i, j, k \in \mathbb{N}$. Hence $a^{i} m \in N$ or $b^{j} m \in N$ or $(a b)^{k} \in(N: M)$. This implies that $N$ is an AG2-absorbing submodule of $M$.

Corollary 1 Let $N$ be a submodule of $M$. Then $N$ is an AG2-absorbing submodule of $M$ if and only if the zero submodule of the R-module $M / N$ is AG2-absorbing.

Corollary 2 Let $I$ and $J$ be ideals of $R$ with $J \subseteq I$. Then $I$ is an AG2-absorbing ideal of $R$ if and only if $I / J$ is an AG2-absorbing ideal of the ring $R / J$.

If we consider Proposition 1 (Corollary 2) in the case of weakly 2-absorbing submodules (ideals), then only the sufficient condition is obtained as follows.

Proposition 2 Let $N$ and $K$ be submodules of $M$ with $K \subseteq N$. If $N$ is a weakly AG2-absorbing submodule of $M$, then $N / K$ is a weakly AG2-absorbing submodule of the R-module $M / K$.

In particular, let $I$ and $J$ be ideals of $R$ with $J \subseteq I$. If I is a weakly AG2-absorbing ideal of $R$, then $I / J$ is a weakly AG2-absorbing ideal of the ring $R / J$.

Proof: Suppose that $N$ is a weakly AG2-absorbing submodule of $M$. Then $N / K$ is a proper submodule of the $R$-module $M / K$. Let $a, b \in R$ and $m \in M$ be such that $0 \neq a b(m+K) \in N / K$. Then $a b m \notin K$. Thus $a b m \neq 0$ so the result follows at once.

However, the necessary condition for Proposition 1 (Corollary 2 ) in the case of weakly 2 -absorbing submodules (ideals) does not hold, as is seen in the following example.
Example 3 Consider $M=R=\mathbb{Z}, N=180 \mathbb{Z}$ and $K=5 \mathbb{Z}$. Then $N / K$ can be viewed as $36 \mathbb{Z}=4 \mathbb{Z} \cap 9 \mathbb{Z}$ which is the intersection of primary submodules. Thus $N / K$ is an AG2-absorbing submodule so that it is weakly AG2-absorbing. Nevertheless, the submodule $N$ is not weakly AG2-absorbing since $0 \neq 2^{2}$. $3^{2} \cdot 5 \in 180 \mathbb{Z}=N$ but $\left(2^{2}\right)^{i} 5 \notin 180 \mathbb{Z},\left(3^{2}\right)^{j} 5 \notin 180 \mathbb{Z}$ and $\left(2^{2} \cdot 3^{2}\right)^{k} \notin 180 \mathbb{Z}$ for all $i, j, k \in \mathbb{N}$.

Knowing that AG2-absorbing submodules are weakly AG2-absorbing submodules, submodules that are weakly AG2-absorbing but not AG2-absorbing should be taken into account. We found that an almost generalized-triple-zero or an $A G$-triplezero is a handy tool for this matter. This notion is analogous to triple-zeros given in Ref. 1 .

Definition 6 Let $N$ be a weakly AG2-absorbing submodule of $M$. An element $(a, b, m) \in R \times R \times M$ is called an almost generalized-triple-zero or an $A G$ triple-zero of $N$ if $a b m=0, a^{i} m \notin N, b^{j} m \notin N$ and $(a b)^{k} \notin(N: M)$ for all $i, j, k \in \mathbb{N}$.

Let $I$ be a weakly AG2-absorbing ideal of $R$. An element $(a, b, c) \in R \times R \times R$ is called an almost generalized-triple-zero or an AG-triple-zero of $I$ if $a b c=0, a^{i} c \notin I, \quad b^{j} c \notin I$ and $(a b)^{k} \notin I$ for all $i, j, k \in \mathbb{N}$.

Proposition 3 If $N$ is a weakly AG2-absorbing submodule of $M$ which is not AG2-absorbing, then $N$ has an $A G$-triple-zero.

If $I$ is a weakly AG2-absorbing ideal of $R$ which is not AG2-absorbing, then I has an AG-triple-zero.

Proof: The proof is obtained directly from the definitions of a weakly AG2-absorbing submodule (ideal) and an AG2-absorbing submodule (ideal).

Some properties of $A G$-triple-zeros which will be used later are provided.

Proposition 4 If $(a, b, m)$ is an AG-triple-zero of $a$ weakly AG2-absorbing submodule $N$ of $M$, then (i) $a b N=0$ (ii) $(N: M) b m=a(N: M) m=(N$ : $M) a m=b(N: M) m=0$ (iii) $(N: M) b N=a(N:$ $M) N=(N: M) a N=b(N: M) N=0$ (iv) $(N:$ $M)^{2} m=0\left(\right.$ v) $(N: M)^{2} N=0$.

Proof: Assume that $(a, b, m)$ is an $A G$-triple-zero of a weakly AG2-absorbing submodule $N$ of $M$.

(i) Suppose that $a b n \neq 0$ for some $n \in N$. Then $0 \neq a b(m+n) \in N$. Since $(a b)^{k} \notin(N: M)$ for all $k \in \mathbb{N}$, we conclude that either $a^{i}(m+n) \in N$ or $b^{j}(m+n) \in N$ for some $i, j \in \mathbb{N}$, and hence $a^{i} m \in N$ or $b^{j} m \in N$. This is a contradiction. Thus $a b N=0$. 
(ii) Suppose that $r b m \neq 0$ for some $r \in(N: M)$. Then $0 \neq(a+r) b m \in N$. Since $b^{j} m \notin N$ for all $j \in \mathbb{N}$, we conclude that either $(a+r)^{p} b^{p} \in$ $(N: M)$ or $(a+r)^{q} m \in N$ for some $p, q \in \mathbb{N}$, and hence by the Binomial theorem, $(a b)^{p}=a^{p} b^{p} \in$ $(N: M)$ or $a^{q} m \in N$, a contradiction. Thus $(N$ : $M) b m=0$. Similarly, one can show that $a(N$ : $M) m=0$. The other results are obtained from the commutativity of $R$.

(iii) Suppose that $r b n \neq 0$ for some $r \in(N: M)$ and $n \in N$. It follows from (i) and (ii) that $0 \neq r b n=$ $(a+r) b(n+m) \in N$. Hence $(a+r)^{i}(n+m) \in N$ or $b^{j}(n+m) \in N$ or $(a+r)^{k} b^{k} \in(N: M)$ for some $i, j, k \in \mathbb{N}$. Thus $a^{i} m \in N$ or $b^{j} m \in N$ or $(a b)^{k} \in(N: M)$ which is a contradiction. Hence $(N: M) b N=0$. Similarly, one can show that $a(N: M) N=0$. The commutativity of $R$ leads to the other results.

(iv) Suppose that $r s m \neq 0$ for some $r, s \in(N: M)$. We obtain from (ii) that $0 \neq r s m=(a+r)(b+$ $s) m \in N$. Hence either $(a+r)^{i} m \in N$ or $(b+$ $s)^{j} m \in N$ or $(a+r)^{k}(b+s)^{k} \in(N: M)$ for some $i, j, k \in \mathbb{N}$. Thus $a^{i} m \in N$ or $b^{j} m \in N$ or $(a b)^{k} \in$ $(N: M)$ which is a contradiction. Hence $(N$ : $M)^{2} m=0$.

(v) Suppose that $r s n \neq 0$ for some $r, s \in(N: M)$ and $n \in N$. It follows from (i)-(iv) that $0 \neq r s n=$ $(r+a)(s+b)(n+m) \in N$. Hence $(r+a)^{i}(n+m) \in$ $N$ or $(s+b)^{j}(n+m) \in N$ or $(r+a)^{k}(s+b)^{k} \in(N$ : $M$ ) for some $i, j, k \in \mathbb{N}$. Thus $a^{i} m \in N$ or $b^{j} m \in$ $N$ or $(a b)^{k} \in(N: M)$ which is a contradiction. Hence $(N: M)^{2} N=0$.

Corollary 3 If $(a, b, c)$ is an AG-triple-zero of a weakly AG2-absorbing ideal $I$ of $R$, then (i) $a b I=$ $a I c=I b c=0$ (ii) $a I^{2}=b I^{2}=c I^{2}=0$ (iii) $I^{3}=0$.

For the rest of this section, we provide some results on AG2-absorbing (weakly AG2-absorbing) ideals that are not parallel to those in terms of submodules.

Proposition 5 If $I$ is an AG2-absorbing ideal of $R$, then $\sqrt{I}$ is an AG2-absorbing ideal of $R$.

Proof: Assume that $I$ is an AG2-absorbing ideal of $R$. Let $a, b, c \in R$ be such that $a b c \in \sqrt{I}$. Thus $(a b c)^{k} \in$ $I$ for some $k \in \mathbb{N}$. Then $a^{k} b^{k} c^{k} \in I$. Hence $\left(a^{k}\right)^{l} c^{k} \in$ $I$ or $\left(b^{k}\right)^{m} c^{k} \in I$ or $\left(a^{k} b^{k}\right)^{n} \in I$ for some $l, m, n \in$ $\mathbb{N}$. Hence we show that $\left(a^{l} c\right)^{k} \in I$ or $\left(b^{m} c\right)^{k} \in I$ or $\left[(a b)^{n}\right]^{k} \in I$, i.e., we have $a^{l} c \in \sqrt{I}$ or $b^{m} c \in \sqrt{I}$ or $(a b)^{n} \in \sqrt{I}$. Hence $\sqrt{I}$ is an AG2-absorbing ideal of $R$.
In the case of weakly AG2-absorbing ideals, we also obtain the analogous result, but only for integral domains.

Proposition 6 Let $R$ be an integral domain. If $I$ is a weakly AG2-absorbing ideal of $R$, then $\sqrt{I}$ is a weakly AG2-absorbing ideal of $R$.

Proof: Assume that $I$ is a weakly AG2-absorbing ideal of an integral domain $R$. Let $a, b, c \in R$ be such that $0 \neq a b c \in \sqrt{I}$. Thus $(a b c)^{k} \in I$ for some $k \in \mathbb{N}$. If $(a b c)^{k}=0$, then $a b c=0$ which is a contradiction. Then $0 \neq a^{k} b^{k} c^{k} \in I$. Hence the result follows in a similar way to the proof of Proposition 5.

Recall that if $I$ is a primary ideal of a ring $R$, then $(I: a)=\{x \in R \quad \mid a x \in I\}$ is also a primary ideal of $R$ for any $a \in R \backslash I$. This result also holds for AG2-absorbing ideals.

Proposition 7 If I is an AG2-absorbing ideal of $R$, then $(I: a)$ is also an AG2-absorbing ideal of $R$ for any $a \in R \backslash I$.

Proof: Assume that $I$ is an AG2-absorbing ideal of $R$. Let $a \in R \backslash I$. Then $(I: a) \neq R$. Moreover, let $b_{1}, b_{2}, b_{3} \in R$ be such that $b_{1} b_{2} b_{3} \in(I: a)$. Then $b_{1} b_{2}\left(b_{3} a\right)=a b_{1} b_{2} b_{3} \in I$ so that $b_{1}^{i} b_{3} a \in I$ or $b_{2}^{j} b_{3} a \in I$ or $\left(b_{1} b_{2}\right)^{k} \in I$ for some $i, j, k \in \mathbb{N}$. Hence $b_{1}^{i} b_{3} \in(I: a)$ or $b_{2}^{j} b_{3} \in(I: a)$ or $\left(b_{1} b_{2}\right)^{k} \in I \subseteq(I:$ $a)$. Hence $(I: a)$ is an AG2-absorbing ideal of $R$ for any $a \in R \backslash I$.

\section{SOME RELATED RESULTS IN MULTIPLICATION MODULES}

In this section, we are concerned with a specific type of modules. We provide some results and some relations regarding AG2-absorbing (weakly AG2absorbing) submodules of a multiplication module including some restricted to the special case of a cyclic $R$-module.

Definition 7 [Ref. 5] A unitary $R$-module $M$ is called a multiplication $R$-module if for each submodule $N$ of $M$, there exists an ideal $I$ of $R$ such that $N=I M$. In general, one can take $N=(N: M) M$ for any submodule $N$ of a multiplication module $M$.

Let $N$ and $K$ be submodules of a multiplication $R$-module $M$ with $N=I_{1} M$ and $K=I_{2} M$ for some ideals $I_{1}$ and $I_{2}$ of $R$. The product of $N$ and $K$, denoted by $N K$, is defined to be $I_{1} I_{2} M$.

The product of the submodules $N=I_{1} M$ and $K=I_{2} M$ of a multiplication $R$-module $M$ can be shown to be independent of the choices of ideals 
$I_{1}$ and $I_{2}$ of $R$. For each integer $k \geqslant 2$, we define $N^{k}=N N^{k-1}$ recursively. Hence $N^{k}=(N: M)^{k} M$ for all $k \in \mathbb{N}$.

Proposition 8 Let $M$ be a multiplication R-module. If $N$ is a weakly AG2-absorbing submodule of $M$ but not AG2-absorbing, then $N^{3}=0$.

Proof: The proof follows immediately from Proposition 4 and the assumption that $M$ is a multiplication $R$-module as follows:

$$
\begin{array}{r}
N^{3}=(N: M)^{3} M=(N: M)^{2}(N: M) M \\
=(N: M)^{2} N=0 .
\end{array}
$$

Proposition 9 Let $M$ be a faithful multiplication $R$-module. If $N$ is a weakly AG2-absorbing submodule of $M$ that is not AG2-absorbing, then $N \subseteq \sqrt{0} M$.

Proof: Recall that $(N: M)^{3} M \subseteq(N: M)^{2} N$ and $(N: M)^{2} N=0$. Then we show that $(N: M)^{3} \subseteq$ $\left((N: M)^{2} N: M\right)=(0: M)$. Moreover, $(0: M)=0$ because $M$ is faithful. Thus $(N: M) \subseteq \sqrt{0}$. Hence $N=(N: M) M \subseteq \sqrt{0} M$.

Recall that an element $a$ of a multiplication module $M$ is said to be nilpotent if $a^{k}=0$ for some $k \in \mathbb{N}$, see Ref. 2. The set of all nilpotent elements of $M$ is denoted by $\operatorname{Nil}(M)$. In particular, it is obvious that a commutative ring $R$ with identity is a multiplication $R$-module and the set of all nilpotent elements of the $R$-module $R$, which is $\operatorname{Nil}(R)$, is simply the standard set of nilpotent elements of the ring $R$.

Corollary 4 If $N$ is a weakly AG2-absorbing but not AG2-absorbing submodule of a multiplication R-module $M$, then $N \subseteq \operatorname{Nil}(M)$.

Towards the end of this section, we provide some relationships between AG2-absorbing (weakly AG2-absorbing) submodules of $M$ and AG2-absorbing (weakly AG2-absorbing) ideals of $R$. However, these results require that $M$ be a cyclic $R$-module. It is known that if $M$ is a cyclic $R$-module with $M=R m$ for some $m \in M$, then $M$ is a multiplication $R$-module and $(N: M)=(N: m)$ for any submodule $N$ of $M$.

Proposition 10 Let $M$ be a cyclic R-module and $N$ a submodule of $M$. (i) The submodule $N$ of $M$ is AG2-absorbing if and only if the ideal $(N: M)$ of $R$ is AG2-absorbing. (ii) If $(N: M)$ is a weakly
AG2-absorbing ideal of $R$, then $N$ is a weakly AG2absorbing submodule of $M$. (iii) If $M$ is faithful and $N$ is weakly AG2-absorbing, then $(N: M)$ is a weakly AG2-absorbing ideal of $R$.

Proof: Let $m \in M$ be such that $M=R m$. Then $(N$ : $M)=(N: m)$. (i) First, assume that $N$ is an AG2-absorbing submodule of $M$. Let $a, b, c \in R$ be such that $a b c \in(N: M)=(N: m)$. Thus $a b c m \in N$. Suppose that $a^{i} c \notin(N: m)$ and $b^{j} c \notin(N: m)$ for all $i, j \in \mathbb{N}$. Hence $a^{i} c m \notin N$ and $b^{j} c m \notin N$ for all $i, j \in \mathbb{N}$. Since $N$ is AG2-absorbing, $(a b)^{k} \in(N: m)=(N: M)$ for some $k \in \mathbb{N}$. Hence $(N: M)$ is an AG2-absorbing ideal of $R$.

Conversely, assume that $(N: M)$ is an AG2-absorbing ideal of $R$. Let $a, b \in R$ and $m^{\prime} \in M$ be such that $a b m^{\prime} \in N$. Thus there exists $r \in R$ with $m^{\prime}=$ $r m$. This yields $a b r m=a b m^{\prime} \in N$. Consequently, $a b r \in(N: m)$. Since $(N: m)$ is an AG2-absorbing ideal, $a^{i} r \in(N: m)$ or $b^{j} r \in(N: m)$ or $(a b)^{k} \in(N$ : $m$ ) for some $i, j, k \in \mathbb{N}$. Hence $a^{i} r m \in N$ or $b^{j} r m \in$ $N$ or $(a b)^{k} \in(N: m)$. It follows that $a^{i} m^{\prime} \in N$ or $b^{j} m^{\prime} \in N$ or $(a b)^{k} \in(N: M)$ as desired.

(ii) Assume that $(N: M)$ is a weakly AG2-absorbing ideal of $R$. Let $a, b \in R$ and $m^{\prime} \in M$ be such that $0 \neq a b m^{\prime} \in N$. Thus there exists $r \in R$ with $m^{\prime}=r m$. Hence $0 \neq a b r m \in N$. Then $0 \neq a b r \in$ $(N: m)$ for otherwise $a b r m=0$, a contradiction. Now a similar argument to the one in the proof of (i) completes this proof.

(iii) Assume that $M$ is faithful and $N$ is a weakly AG2-absorbing submodule of $M$. Let $a, b, c \in R$ be such that $0 \neq a b c \in(N: M)=(N: m)$ but $a^{i} b \notin(N: m)$ and $b^{j} c \notin(N: m)$ for all $i, j \in \mathbb{N}$. Then $0 \neq a b c m \in N$ for otherwise $a b c m=0$ implies that $a b c \in(0: m)=(0: M)=0$, a contradiction. Again, a similar argument to the one in the proof of (i) completes this proof.

Example 4 Consider $M=\mathbb{Z}_{72}$ as a $\mathbb{Z}$-module. Let $N=\langle\overline{36}\rangle$. Then $(N: M)=36 \mathbb{Z}=4 \mathbb{Z} \cap 9 \mathbb{Z}$ is an AG2absorbing ideal of $\mathbb{Z}$. Hence, by Proposition 10 , we show that $N$ is an AG2-absorbing submodule of $M$.

Corollary 5 Let $M$ be a faithful cyclic R-module and $N$ a submodule of $M$. Then $N$ is a weakly AG2-absorbing submodule of $M$ if and only if $(N: M)$ is a weakly AG2-absorbing ideal of $R$.

\section{RINGS WITH THE PROPERTY THAT ALL PROPER IDEALS ARE WEAKLY AG2-ABSORBING}

In this section, regular rings with the property that all proper ideals are weakly AG2-absorbing are investigated. We will provide some conditions on 
regular rings which are equivalent to all proper ideals being weakly AG2-absorbing.

Definition 8 An element $a$ of a ring (not necessarily commutative with identity) $R$ is said to be von Neumann regular if there exists an element $x$ of $R$ such that $a x a=a$. A ring (not necessarily commutative with identity) $R$ is said to be von Neumann regular if each element of $R$ is von Neumann regular.

If $R$ is a commutative von Neumann regular ring and $a, b, c \in R$, then one can show that $a b c=0$ if and only if $a^{i} b^{j} c^{k}=0$ for some $i, j, k \in \mathbb{N}$. For a commutative ring $R$ with identity 1 , let $J(R)$ denote the Jacobson radical, i.e., the intersection of all maximal ideals of $R$. Note that $x \in J(R)$ if and only if $1-r x$ is a unit for all $r \in R$.

Proposition 11 Let $R$ be a commutative von Neumann regular ring with identity and $a, b, c \in J(R)$. Then the ideal abcR is weakly AG2-absorbing if and only if $a b c=0$.

Proof: If $a b c=0$, then it is obvious that $a b c R$ is a weakly AG2-absorbing ideal of $R$. Now suppose that $a b c \neq 0$ and $a b c R$ is a weakly AG2-absorbing ideal of $R$. Thus $a^{i} b^{j} c^{k} \neq 0$ for all $i, j, k \in \mathbb{N}$. Since $a b c \in$ $a b c R \backslash\{0\}$, we conclude that either $a^{i} c \in a b c R$ or $b^{j} c \in a b c R$ or $(a b)^{k} \in a b c R$ for some $i, j, k \in \mathbb{N}$. First, we assume that $a^{i} c \in a b c R$. Since $R$ is a commutative von Neumann regular ring, $a b c R=$ $\left(a^{i} x\right) b c R=a^{i} b c x R \subseteq a^{i} b c R$ for some $x \in R$. Thus $a^{i} c=a^{i} b c d$ for some $d \in R$, and hence $a^{i} c(1-b d)=$ 0 . Since $b \in J(R)$, it follows that $b d \in J(R)$ so that $1-b d$ is a unit of $R$. Thus $a^{i} c(1-b d)=0$ implies that $a^{i} c=0$, and thus $a^{i} b c=0$ which is a contradiction. Similarly, the other two cases are not possible. Hence $a b c=0$.

Corollary 6 Let $R$ be a commutative von Neumann regular ring with identity, $n \in \mathbb{N}$ and $a_{1}, a_{2}, \ldots, a_{n} \in$ $J(R)$. Then the ideal $a_{1} a_{2} \cdots a_{n} R$ is weakly AG2-absorbing if and only if $a_{1} a_{2} \cdots a_{n}=0$.

Definition 9 A commutative ring $R$ with identity is called a local ring if it satisfies any one of the following equivalent conditions: (a) $R$ has a unique maximal ideal. (b) $1 \neq 0$ and the sum of any two non-units of $R$ is a non-unit. (c) $1 \neq 0$ and if $x$ is any element of $R$, then $x$ or $1-x$ is a unit.

Note that some authors require that local rings be Noetherian, and non-Noetherian rings are then called quasi local rings. In this work, $R$ need not be Noetherian. Since a local ring $R$ has a unique maximal ideal, say $K$, we denote this local ring $R$ by $(R, K)$. Observe that in a local ring $(R, K)$, its Jacobson radical is $K$, i.e., $J(R)=K$. Moreover, if $a \in R \backslash K$, then $a$ is a unit of $R$.

Proposition 12 Let $(R, K)$ be a commutative von Neumann regular local ring. Then the following are equivalent. (i) Every proper ideal of $R$ is weakly AG2absorbing. (ii) $K^{3}=0$. (iii) $K^{n}=0$ for all $n \in \mathbb{N}$. (iv) $K^{n}=0$ for some $n \in \mathbb{N}$.

Proof: (i) $\Leftrightarrow$ (ii). Assume that every proper ideal of $R$ is weakly AG2-absorbing. Let $a, b, c \in K$. Since $a b c R$ is a proper ideal of $R$, by (i) $a b c R$ is weakly AG2-absorbing, so that $a b c=0$ by Proposition 11 . Thus $K^{3}=0$. Conversely, assume that $K^{3}=0$ and let $N$ be a proper ideal of $R$ such that $N \neq 0$. Suppose that $a b c \in N$ and $a b c \neq 0$. Since $K^{3}=0$ and $a b c \neq$ 0 , we show that $a \notin K$ or $b \notin K$ or $c \notin K$. But $(R, K)$ is a von Neumann regular local ring, thus $a$ or $b$ or $c$ is a unit of $R$ and then either $b c \in N$ or $a c \in N$ or $a b \in N$. Hence $N$ is a weakly AG2-absorbing ideal of $R$.

(ii) $\Leftrightarrow$ (iii). Since $R$ is a regular ring, we obtain this part immediately: For each $n \in \mathbb{N}$,

$$
K \subseteq K R K \subseteq K^{2} \subseteq(K R K) K \subseteq K^{3} \subseteq \cdots \subseteq K^{n} \subseteq K .
$$

(iii) $\Leftrightarrow$ (iv). This follows directly from (1).

It is clear that any field $F$ is a von Neumann regular local ring $(F,\{0\})$.

Corollary 7 Any commutative von Neumann regular local ring $(R, K)$ in which every proper ideal is weakly AG2-absorbing must be a field.

Proof: It follows from Proposition 12 that $K=0$. Thus the only ideals of $R$ are $\{0\}$ and $R$ itself. Hence $R$ is a field.

The next theorem provides a characterization of commutative von Neumann regular local rings in which every proper ideal is AG2-absorbing.

Theorem 2 Let $(R, K)$ be a commutative von Neumann regular local ring. Then $K^{2}=0$ if and only if every proper ideal of $R$ is AG2-absorbing.

Proof: Assume that $K^{2}=0$. Let $J$ be a proper ideal of $R$ and $a, b, c \in R$ be such that $a b c \in J$. Note that $J$ is a weakly AG2-absorbing ideal of $R$ by Proposition 12 . Hence if $0 \neq a b c \in J$, then there is nothing to prove. Thus assume that $a b c=0$ so that $a b c \in K$. We divide our argument into 2 cases. Case 1: $a$ or $b$ or $c$ is a unit. Since $a b c=0$, it 
follows that $b c=0$ or $a c=0$ or $a b=0$. Case 2: $a, b$ and $c$ are not units. Since $R$ is local, $a, b, c \in K$. Thus $a c=b c=a b=0$ from $K^{2}=0$. Thus either $a b=0 \in J$ or $b c=0 \in J$ or $a c=0 \in J$. Hence $J$ is an AG2-absorbing ideal of $R$.

Conversely, assume that every proper ideal of $R$ is an AG2-absorbing ideal. Since an AG2-absorbing ideal is also a weakly AG2-absorbing ideal of $R$, by Proposition 12 we show that $K^{2}=0$ as desired.

Proposition 12, Corollary 7 and Theorem 2 provide conditions that make a commutative von Neumann regular local ring be a field. This significant result can be stated as follows.

Theorem 3 Let $R$ be a commutative ring with identity. Then the following are equivalent. (i) $R$ is a field. (ii) $R$ is a commutative von Neumann regular local ring in which every proper ideal is weakly AG2absorbing. (iii) $R$ is a commutative von Neumann regular local ring in which every proper ideal is AG2absorbing.

Hence commutative von Neumann regular local rings with the property that all proper ideals are weakly AG2-absorbing must be fields. In such a ring, an ideal is AG2-absorbing if and only if it is weakly AG2-absorbing, in which case it is, of course, the zero ideal.

Corollary 8 Let $R$ be a commutative von Neumann regular local ring with identity which is not a field. Then there exist proper ideals $I \neq\{0\}$ and $J$ of $R$, not necessary distinct, such that the ideal I is not weakly AG2-absorbing and the ideal $J$ is not AG2-absorbing.

\section{PROPERTIES OF AG2-ABSORBING IDEALS AND WEAKLY AG2-ABSORBING IDEALS OF DECOMPOSABLE COMMUTATIVE RINGS}

A commutative ring $R$ is said to be decomposable if it can be written as a product of commutative non-zero rings, i.e., $R=R_{1} \times R_{2} \times \cdots \times R_{n}$ for some commutative non-zero rings $R_{1}, R_{2}, \ldots, R_{n}$.

Proposition 13 Let $R=R_{1} \times R_{2}$ be a decomposable commutative ring where each of $R_{1}$ and $R_{2}$ is a commutative ring with identity and I is a proper ideal of $R_{1}$. The following statements are equivalent. (i) $I \times R_{2}$ is a weakly AG2-absorbing ideal of $R$. (ii) $I \times R_{2}$ is an AG2-absorbing ideal of $R$. (iii) $I$ is an AG2-absorbing ideal of $R_{1}$.

Proof: (i) $\Rightarrow$ (ii). Assume that $I \times R_{2}$ is a weakly AG2absorbing ideal of $R$. Suppose that $I \times R_{2} \subseteq \operatorname{Nil}(R)$. Then for each $r \in R_{2}$ there exists $k \in \mathbb{N}$ such that $r^{k}=0$. In particular, $1^{k}=0$ which is a contradiction. Thus $I \times R_{2}$ is a weakly AG2-absorbing ideal of $R$ such that $I \times R_{2} \nsubseteq \operatorname{Nil}(R)$. By Corollary 4, the ideal $I \times R_{2}$ must be AG2-absorbing.

(ii) $\Rightarrow$ (iii). Assume that $I \times R_{2}$ is an AG2-absorbing ideal of $R$. Let $a, b, c \in R_{1}$ be such that $a b c \in I$. Thus $(a, 0)(b, 0)(c, 0) \in I \times R_{2}$, so $(a, 0)^{i}(c, 0) \in I \times R_{2}$ or $(b, 0)^{j}(c, 0) \in I \times R_{2}$ or $[(a, 0)(b, 0)]^{k} \in I \times R_{2}$ for some $i, j, k \in \mathbb{N}$. Then $\left(a^{i} c, 0\right) \in I \times R_{2}$ or $\left(b^{j} c, 0\right) \in$ $I \times R_{2}$ or $\left((a b)^{k}, 0\right) \in I \times R_{2}$. Hence $a^{i} c \in I$ or $b^{j} c \in I$ or $(a b)^{k} \in I$. Hence the result is obtained.

(iii) $\Rightarrow$ (i). Assume that $I$ is an AG2-absorbing ideal of $R_{1}$. Let $(a, b),(c, d),(e, f) \in R$ be such that $(a, b)(c, d)(e, f) \in I \times R_{2} \backslash\{(0,0)\}$. Then ace $\in I$. Thus $a^{i} e \in I$ or $c^{j} e \in I$ or $(a c)^{k} \in I$ for some $i, j, k \in$ $\mathbb{N}$. Hence $(a, b)^{i}(e, f) \in I \times R_{2}$ or $(c, d)^{j}(e, f) \in I \times R_{2}$ or $[(a, b)(c, d)]^{k} \in I \times R_{2}$ as desired.

As a result, any ideal of the form $I \times R_{2}$ (where $I$ is a proper ideal of $R_{1}$ ) is AG2-absorbing if and only if it is weakly AG2-absorbing, and in this case $I$ must be an AG2-absorbing ideal of $R_{1}$.

The next proposition gives conditions under which AG2-absorbing ideals and weakly AG2-absorbing ideals of decomposable commutative rings coincide.

Proposition 14 Let $R=R_{1} \times R_{2}$ be a decomposable commutative ring where each of $R_{1}$ and $R_{2}$ is a commutative ring with identity. Moreover, let I be a non-zero proper ideal of $R_{1}$ and $J$ a non-zero ideal of $R_{2}$. The following statements are equivalent. (i) $I \times J$ is a weakly AG2-absorbing ideal of $R$. (ii) $J=R_{2}$ and $I$ is an AG2-absorbing ideal of $R_{1}$, or both of $I$ and $J$ are primary ideals. (iii) $I \times J$ is an AG2-absorbing ideal of $R$.

Proof: (i) $\Rightarrow$ (ii). Assume that $I \times J$ is a weakly AG2absorbing ideal of $R$. If $J=R_{2}$, then $I$ is an AG2absorbing ideal of $R_{1}$ by Proposition 13. Suppose that $J \neq R_{2}$. To show that $J$ is a primary ideal of $R_{2}$, let $a, b \in R_{2}$ be such that $a b \in J$ and let $0 \neq l \in I$. Then $(1, a)(l, 1)(1, b)=(l, a b) \in I \times J \backslash\{(0,0)\}$. Since $(1, a)^{i}(1, b)=\left(1, a^{i} b\right) \notin I \times J$ for all $i \in \mathbb{N}$, we have $\left(l^{j}, b\right)=(l, 1)^{j}(1, b) \in I \times J$ or $\left(l^{k}, a^{k}\right)=$ $[(1, a)(l, 1)]^{k} \in I \times J$ for some $j, k \in \mathbb{N}$. Thus $b \in J$ or $a^{k} \in J$. Hence $J$ is a primary ideal of $R_{2}$. Similarly, $I$ is a primary ideal of $R_{1}$.

(ii) $\Rightarrow$ (iii). Assume (ii) holds. If $J=R_{2}$ and $I$ is an AG2-absorbing ideal of $R_{1}$, then $I \times R_{2}$ is an AG2-absorbing ideal of $R$ by Proposition 13 . Suppose that both $I$ and $J$ are primary ideals. Moreover, let $a_{1}, a_{2}, a_{3} \in R_{1}$ and $b_{1}, b_{2}, b_{3} \in R_{2}$ be such that $\left(a_{1}, b_{1}\right)\left(a_{2}, b_{2}\right)\left(a_{3}, b_{3}\right) \in I \times J$. Thus 
$\left(a_{1} a_{2} a_{3}, b_{1} b_{2} b_{3}\right) \in I \times J$. Since $I$ and $J$ are primary ideals, at least one $a_{i}^{p_{i}}$ is in $I$ for some $p_{i} \in \mathbb{N}$, say $a_{1}^{p_{1}}$, and at least one $b_{i}^{q_{i}}$ is in $J$ for some $q_{i} \in$ $\mathbb{N}$. If $b_{1}^{q_{1}} \in J$, then $\left(a_{1}, b_{1}\right)^{p_{1}+q_{1}} \in I \times J$ so that $\left[\left(a_{1}, b_{1}\right)\left(a_{2}, b_{2}\right)\right]^{p_{1}+q_{1}} \in I \times J$. Otherwise, without loss of generality, assume that $b_{2}^{q_{2}} \in J$. Hence $\left[\left(a_{1}, b_{1}\right)\left(a_{2}, b_{2}\right)\right]^{p_{1}+q_{2}} \in I \times J$. Hence $I \times J$ is an AG2absorbing ideal of $R$.

(iii) $\Rightarrow$ (i). This is clear.

Now, we consider weakly AG2-absorbing but not AG2-absorbing ideals. The next result gives their characterization in decomposable commutative rings.

Proposition 15 Let $R=R_{1} \times R_{2}$ be a decomposable commutative ring where each of $R_{1}$ and $R_{2}$ is a commutative ring with identity. Let I be a non-zero proper ideal of $R_{1}$ and $J$ an ideal of $R_{2}$. The following statements are equivalent. (i) $I \times J$ is a weakly AG2absorbing but not AG2-absorbing ideal of $R$. (ii) $I$ is a weakly primary but not primary ideal of $R_{1}$ and $J=\{0\}$ is a primary ideal of $R_{2}$.

Proof: (i) $\Rightarrow$ (ii). Assume that $I \times J$ is a weakly AG2absorbing but not AG2-absorbing ideal of $R$. If $J \neq$ $\{0\}$, then $I \times J$ is an AG2-absorbing ideal of $R$ by Proposition 14, contradicting the hypothesis. Thus $J=\{0\}$. Next, we show that $J=\{0\}$ is a primary ideal of $R_{2}$. Let $a, b \in R_{2}$ be such that $a b \in\{0\}$ and let $0 \neq l \in I$. Then $(1, a)(l, 1)(1, b)=(l, a b) \in I \times$ $\{0\} \backslash\{(0,0)\}$. Since $(1, a)^{i}(1, b)=\left(1, a^{i} b\right) \notin I \times\{0\}$ for all $i \in \mathbb{N}$, we have $\left(l^{j}, b\right)=(l, 1)^{j}(1, b) \in I \times\{0\}$ or $\left(l^{k}, a^{k}\right)=[(1, a)(l, 1)]^{k} \in I \times\{0\}$ for some $j, k \in \mathbb{N}$. Hence $b \in\{0\}$ or $a^{k} \in\{0\}$. Thus $\{0\}$ is a primary ideal of $R_{2}$. Now, to show that $I$ is a weakly primary ideal of $R_{1}$, let $a, b \in R_{1}$ be such that $a b \in I \backslash\{0\}$. Since $(a, 1)(1,0)(b, 1)=(a b, 0) \in I \times\{0\} \backslash\{(0,0)\}$ and $(a, 1)^{i}(b, 1)=\left(a^{i} b, 1\right) \notin I \times\{0\}$ for all $i \in \mathbb{N}$, we conclude that either $(b, 0)=(1,0)^{j}(b, 1) \in I \times\{0\}$ or $\left(a^{k}, 0\right)=[(a, 1)(1,0)]^{k} \in I \times\{0\}$ for some $j, k \in \mathbb{N}$. Thus either $b \in I$ or $a^{k} \in I$. Hence $I$ is a weakly primary ideal of $R_{1}$.

Finally, suppose that $I$ is a primary ideal of $R_{1}$. We claim that $I \times\{0\}$ is an AG2-absorbing ideal of $R$. Let $(a c e, b d f)=(a, b)(c, d)(e, f) \in I \times\{0\}$. Since $I$ and $\{0\}$ are primary ideals of $R_{1}$ and $R_{2}$, respectively, we may assume that $a^{r} \in I$ and $b^{s} \in\{0\}$ for some $r, s \in \mathbb{N}$. Thus $[(a, b)(c, d)]^{r+s} \in I \times\{0\}$. Hence $I \times\{0\}$ is an AG2-absorbing ideal of $R$ as claimed which contradicts the assumption. Hence $I$ is a weakly primary but not primary ideal of $R_{1}$ as desired.

(ii) $\Rightarrow$ (i). Assume that $I$ is a weakly primary but not primary ideal of $R_{1}$ and $J=\{0\}$ is a primary ideal of $R_{2}$. Let $a_{1}, a_{2}, a_{3} \in R_{1}$ and $b_{1}, b_{2}, b_{3} \in R_{2}$ be such that $\left(a_{1} a_{2} a_{3}, b_{1} b_{2} b_{3}\right)=$ $\left(a_{1}, b_{1}\right)\left(a_{2}, b_{2}\right)\left(a_{3}, b_{3}\right) \in(I \times J) \backslash\{(0,0)\}=(I \times$ $\{0\}) \backslash\{(0,0)\}$. Thus $a_{1} a_{2} a_{3} \in I \backslash\{0\}$ and $b_{1} b_{2} b_{3} \in$ $\{0\}$. Since $I$ is weakly primary, we may assume $a_{1}^{p_{1}} \in$ $I$ for some $p_{1} \in \mathbb{N}$. Moreover, since $\{0\}$ is a primary ideal of $R_{2}$, there is $j \in\{1,2,3\}$ such that $b_{j}^{q_{j}} \in\{0\}$ for some $q_{j} \in \mathbb{N}$. Hence $\left[\left(a_{1}, b_{1}\right)\left(a_{j}, b_{j}\right)\right]^{p_{1}+q_{j}} \in I \times$ $\{0\}$. Hence $I \times\{0\}=I \times J$ is a weakly AG2-absorbing ideal of $R$. Finally, we show that $I \times\{0\}$ is not an AG2-absorbing ideal of $R$. Since $I$ is a weakly primary ideal of $R_{1}$ but not primary, there are $a$, $b \in R_{1}$ such that $a b=0$ but $a \notin I$ and $b^{m} \notin I$ for all $m \in \mathbb{N}$. Since $(a, 1)(1,0)(b, 1)=(0,0) \in I \times\{0\}$ but

$$
\begin{aligned}
\forall i \in \mathbb{N},(a, 1)^{i}(b, 1) & =\left(a^{i} b, 1\right) \notin I \times\{0\} \text { and } \\
\forall j \in \mathbb{N},(1,0)^{j}(b, 1) & =(b, 0) \notin I \times\{0\} \text { and } \\
\forall k \in \mathbb{N},[(a, 1)(1,0)]^{k} & =\left(a^{k}, 0\right) \notin I \times\{0\},
\end{aligned}
$$

we conclude that $I \times\{0\}$ is not an AG2-absorbing ideal of $R$.

It seems that Proposition 14 and Proposition 15 contradict each other. But, in fact, they do not. The assumptions of these two propositions are different, because $J$ must be a non-zero ideal in Proposition 14 but $J$ is just an ideal in Proposition 15. This means that $J=0$ or $J \neq 0$ are key conditions that distinguish between them. Proposition 15 is a very strong characterization; it ensures that in a decomposable commutative ring an ideal of the form $I \times J$ that is weakly AG2-absorbing but not AG2absorbing (where $I$ is a non-zero proper ideal) can only occur under the condition that $J$ is a non-zero primary ideal.

For the rest of this section, let $R_{1}, R_{2}$ and $R_{3}$ be commutative rings with identities. Recall that an ideal $I$ of $R_{1} \times R_{2} \times R_{3}$ must be of the form $I_{1} \times I_{2} \times I_{3}$ where $I_{1}, I_{2}$ and $I_{3}$ are ideals of $R_{1}, R_{2}$ and $R_{3}$, respectively. The next two theorems show what weakly AG2-absorbing ideals of $R_{1} \times R_{2} \times R_{3}$ look like.

Theorem 4 Let $R=R_{1} \times R_{2} \times R_{3}$ be a decomposable commutative ring. If $I$ is a weakly AG2-absorbing ideal of $R$, then either $I=\{(0,0,0)\}$ or $I$ is an AG2absorbing ideal of $R$.

Proof: Assume that $I$ is a weakly AG2-absorbing ideal of $R$. Since $\{(0,0,0)\}$ is a weakly AG2absorbing ideal of any ring, we may assume that $I_{1} \times I_{2} \times I_{3}=I \neq\{(0,0,0)\}$. Thus there is an element $(0,0,0) \neq(a, b, c)=(a, 1,1)(1, b, 1)(1,1, c) \in$ 
I. Hence $\left(a^{i}, 1, c\right)=(a, 1,1)^{i}(1,1, c) \in I$ or $\left(1, b^{j}, c\right)=(1, b, 1)^{j}(1,1, c) \in I$ or $\left(a^{k}, b^{k}, 1\right)=$ $[(a, 1,1)(1, b, 1)]^{k} \in I$ for some $i, j, k \in \mathbb{N}$. Thus $I_{2}=$ $R_{2}$ or $I_{1}=R_{1}$ or $I_{3}=R_{3}$, respectively. Consequently, $I=I_{1} \times R_{2} \times I_{3}$ or $I=R_{1} \times I_{2} \times I_{3}$ or $I=I_{1} \times I_{2} \times R_{3}$. If $I \subseteq \operatorname{Nil}(R)$, then $R_{1}=\{0\}$ or $R_{2}=\{0\}$ or $R_{3}=\{0\}$, which is a contradiction. Since $I$ is a weakly AG2absorbing ideal of $R$ such that $I \nsubseteq \operatorname{Nil}(R)$, the ideal $I$ must be an AG2-absorbing ideal of $R$.

The above theorem shows that in a ring $R=$ $R_{1} \times R_{2} \times R_{3}$, a weakly AG2-absorbing ideal which is not AG2-absorbing is unique and it must be the zero ideal.

Theorem 5 Let $R=R_{1} \times R_{2} \times R_{3}$ be a decomposable commutative ring, $I_{1}$ a proper ideal of $R_{1}$, and $I_{2}$ and $I_{3}$ ideals of $R_{2}$ and $R_{3}$, respectively, such that $I=I_{1} \times I_{2} \times I_{3} \neq\{(0,0,0)\}$. The following statements are equivalent. (i) $I=I_{1} \times I_{2} \times I_{3}$ is a weakly AG2absorbing ideal of $R$. (ii) $I=I_{1} \times I_{2} \times I_{3}$ is an AG2absorbing ideal of $R$. (iii) $I=I_{1} \times R_{2} \times R_{3}$ and $I_{1}$ is an AG2-absorbing ideal of $R_{1}$ or $I=I_{1} \times I_{2} \times R_{3}$ where $I_{1}$ and $I_{2}$ are primary ideals or $I=I_{1} \times R_{2} \times I_{3}$ where $I_{1}$ and $I_{3}$ are primary ideals.

Proof: (i) $\Rightarrow$ (ii). Assume that $I$ is a weakly AG2absorbing ideal of $R$. Since $I$ is non-zero, $I$ is an AG2-absorbing ideal of $R$ by Theorem 4 .

(ii) $\Rightarrow$ (iii). Assume that $I$ is an AG2-absorbing ideal of $R$. To show that $I_{1}$ is an AG2-absorbing ideal of $R_{1}$, let $a, b, c \in R_{1}$ be such that $a b c \in I_{1}$. Then $(a b c, 0,0)=(a, 0,0)(b, 0,0)(c, 0,0) \in I$ so we show that $a^{i} c \in I_{1}$ or $b^{j} c \in I_{1}$ or $(a b)^{k} \in I_{1}$ for some $i, j, k \in \mathbb{N}$ as desired, because the ideal $I$ is AG2absorbing. Since $I_{1}$ is a proper ideal, and by a proof similar to that of Theorem 4, either $I_{2}=R_{2}$ or $I_{3}=$ $R_{3}$. We separate the remainder of the argument into 3 cases: Case $1 I_{2}=R_{2}$ and $I_{3}=R_{3}$, Case $2 I_{2} \neq R_{2}$ and $I_{3}=R_{3}$, Case $3 I_{2}=R_{2}$ and $I_{3} \neq R_{3}$. The first case leads us to the result that $I=I_{1} \times R_{2} \times R_{3}$ and $I_{1}$ is AG2-absorbing. Next, assume that $I_{2} \neq R_{2}$ and $I_{3}=R_{3}$. In order to show that $I_{1}$ and $I_{2}$ are primary ideals, let $a, b \in R_{1}$ and $c, d \in R_{2}$ be such that $a b \in$ $I_{1}$ and $c d \in I_{2}$. Then $(a, 1,1)(1, c d, 1)(b, 1,1)=$ $(a b, c d, 1) \in I \backslash\{(0,0,0)\}$. Since $(a, 1,1)^{i}(b, 1,1)=$ $\left(a^{i} b, 1,1\right) \notin I$ for all $i \in \mathbb{N}$, it follows that there exist $j, k \in \mathbb{N}$ such that $\left(b,(c d)^{j}, 1\right)=(1, c d, 1)^{j}(b, 1,1) \in$ $I$ or $\left(a^{k},(c d)^{k}, 1\right)=[(a, 1,1)(1, c d, 1)]^{k} \in I$. Thus $b \in I_{1}$ or $a^{k} \in I_{1}$. Hence $I_{1}$ is a primary ideal of $R_{1}$. Similarly, since $(a b, 1,1)(1, c, 1)(1, d, 1)=$ $(a b, c d, 1) \in I \backslash\{(0,0,0)\}$ and $(1, c, 1)^{i}(1, d, 1)=$ $\left(1, c^{i} d, 1\right) \notin I$ for all $i \in \mathbb{N}$, there are $j, k \in \mathbb{N}$ such that $\left((a b)^{j}, d, 1\right)=(a b, 1,1)^{j}(1, d, 1) \in I$ or $\left((a b)^{k}, c^{k}, 1\right)=[(a b, 1,1)(1, c, 1)]^{k} \in I$. Thus $d \in$ $I_{2}$ or $c^{k} \in I_{2}$. Hence $I_{2}$ is a primary ideal of $R_{2}$. Finally, assume that $I_{2}=R_{2}$ and $I_{3} \neq R_{3}$. By an argument similar to the one that we applied to the ideal $I_{1} \times I_{2} \times R_{3}$, we conclude that $I_{1}$ and $I_{3}$ are primary ideals.

(iii) $\Rightarrow$ (i). If $I$ is one of the given three forms, then by applying the proof of (iii) $\Rightarrow$ (i) in Proposition 13, it is easy to verify that $I$ is a weakly AG2-absorbing ideal of $R$ as desired.

Note that Theorem 5 can be applied to decomposable commutative rings of the form $R_{1} \times R_{2} \times \cdots \times$ $R_{m}$ for any integer $m \geqslant 4$ by considering $R_{3} \times \cdots \times R_{m}$ as a single ring.

Acknowledgements: The authors appreciate the comments from the referees which helped to make this manuscript more complete. The first author thanks the 90th anniversary of Chulalongkorn University Fund (Ratchadaphiseksomphot Endowment Fund) for partial support.

\section{REFERENCES}

1. Badawi A, Darani A (2013) On weakly 2-absorbing ideals of commutative rings. Houston J Math 39, 441-52.

2. Darani A, Soheilnia F (2011) 2-absorbing and weakly 2-absorbing submodules. Thai J Math 9, 577-84.

3. Badawi A (2007) On 2-absorbing ideals of commutative rings. Bull Aust Math Soc 75, 417-29.

4. Smith PF (2001) Primary modules over commutative rings. Glasgow Math $J$ 43, 103-11.

5. Smith PF (1988) Some remarks on multiplication modules. Arch Math 50, 223-35. 